\title{
Sistemas silvopastoriles del centro y norte de la Provincia de Misiones, Argentina
}

\author{
Pantiu, A.J.'; Capellari, A. ${ }^{1}$; Kurtz, V.D. ${ }^{2}$ \\ ${ }^{1}$ Facultad de Ciencias Veterinarias, Universidad Nacional del Nordeste, UNNE, Sargento Cabral 2139, \\ Corrientes (3400), Argentina. Tel/Fax 03783-425753. ${ }^{2}$ Agencia de Extensión, INTA Eldorado, Misiones, \\ Argentina. E-mail: acapellari@vet.unne.edu.ar.
}

\begin{abstract}
Resumen
Pantiu, A.J.; Capellari, A.; Kurtz, V.D.: Sistemas silvopastoriles del centro y norte de la Provincia de Misiones, Argentina. Rev. vet. 21: 1, 69-75, 2010. Los sistemas silvopastoriles representan una modalidad de uso de la tierra donde coexisten interacciones ambientales, económicas y sociales entre los componentes arbóreo, forrajero, ganadero, edáfico y humano, bajo un manejo integrado. El objetivo del trabajo fue establecer la producción física de los sistemas silvopastoriles como una opción para mejorar la rentabilidad del recurso tierra a través de la diversificación productiva, con el menor impacto sobre el uso del suelo y medio ambiente. Mediante un estudio de casos se describe el desarrollo de dos sistemas forestoganaderos ubicados en el centro y norte de Misiones, donde se informan variables sobre la producción de carne (categoría animal, biotipo, ganancia de peso total y diaria, carga animal), caracterización de las especies forrajeras (especie, implantación, producción de materia seca, porcentaje de proteína) y caracterización de las especies forestales (especie, longitud de copa, densidad de plantación). Los resultados obtenidos revelan que el estudio de los sistemas silvopastoriles constituye una nueva alternativa para generar modelos productivos más rentables y eficientes en un marco de sustentabilidad, permitiendo obtener dos o más productos en un mismo sitio con menor impacto sobre el uso del suelo y medio ambiente.
\end{abstract}

Palabras clave: bovino, sistemas silvopastoriles, producción de carne.

\begin{abstract}
Pantiu, A.J.; Capellari, A.; Kurtz, V.D.: Survey of silvopastoral systems in central and northern Province of Misiones, Argentina. Rev. vet. 21: 1, 69-75, 2010. Silvopastoral systems represent a form of land use where coexist environmental, economic and social interactions among arboreal components and forage, livestock, soil and human beings, under an integrated management. The objective of this study was to establish the physical production of silvopastoral systems as an option to improve the profitability of land resources by means of diversification, with the least impact on land use and environment. Through a case study we describe the development of two forestal-cattle farm systems located in the center and north of Misiones, northeastern Argentina, where variables are reported on the production of meat (animal category, biotype, total and daily weight gain, stocking rate), characterization of forages (species, implantation, dry matter, protein percent), and characterization of forest species (species, crown length, plantation density). It can be concluded that silvopastoral systems represent a new alternative to generate more profitable and efficient production models in a sustainable framework, that allow to obtain two or more products in the same place with the least impact on the use of soil and environment.
\end{abstract}

Key words: cattle, silvopastoral systems, beef cattle production.

\section{INTRODUCCIÓN}

La Provincia de Misiones se encuentra ubicada en la región nordeste de la República Argentina. Casi la totalidad de sus límites está conformada por ríos, el Iguazú al norte, el Paraná al oeste, el Pepirí Guazú y el Uruguay al este y el Chimiray al sur ${ }^{5}$. Misiones se

Recibido: 12 mayo 2010 / Aceptado: 3 junio 2010 caracteriza por tener un clima subtropical húmedo, con temperaturas medias anuales del orden de $\operatorname{los} 21,5^{\circ} \mathrm{C}$, heladas invernales y precipitaciones relativamente isohigras del orden de los $1.800 \mathrm{~mm}$ anuales ${ }^{7}$.

Los suelos son el resultado de la descomposición del basalto y su aptitud de uso está condicionada por factores tales como fertilidad deficiente, susceptibilidad a la erosión, pedregosidad, acidez, toxicidad de aluminio y exceso de humedad que limita la aireación ${ }^{2}$. A 
su vez, el relieve del terreno presenta ondulaciones con pendientes variables que superan desniveles del 5\% y que en algunos casos son mayores del $12 \%{ }^{5}$. Los suelos de Misiones presentan cierta fragilidad; el $70 \%$ de la superficie tiene fuertes pendientes no aptas para la agricultura, pero sí para la forestación. La profundidad es siempre superior a un metro, por cual es apto para las especies de gran desarrollo radical ${ }^{5}$.

Debido a las características de los suelos de esta provincia, el sector forestal representa la actividad económica más importante y la que genera mayores ingresos ${ }^{7}$. Sin embargo, existe en la actualidad un conjunto de factores que condicionan fuertemente su rentabilidad, como el inadecuado manejo forestal, el cual origina bajos precios por el producto y tiempo de retorno económico prolongado. Estos factores se hacen aún más importantes en el estrato de productores pequeños y medianos ${ }^{14}$. La provincia cuenta aproximadamente con 371.000 ha reforestadas, correspondiendo el $84 \%$ de esta superficie a coníferas y dentro de ellas el $76 \%$ a pinos ${ }^{6}$.

En cuanto a la actividad ganadera, Misiones representa el $0,5 \%$ del stock ganadero del país; comprende una superficie de 350.000 ha y 400.000 cabezas distribuidas en un total de 14.810 productores. El $92 \%$ de los establecimientos posee 1 a 50 cabezas, y contiene el $41 \%$ de las existencias provinciales. El $85 \%$ de estos productores destina el $20 \%$ de la superficie a la ganadería y desarrolla sistemas mixtos de ciclo completo y manejo extensivo ${ }^{6}$.

La ganadería tradicionalmente se practica a cielo abierto, predominando el ganado tipo racial índico, que está siendo paulatinamente reemplazado por cruzas Cebú-Hereford, Braford, Angus y criolla. La carga animal es muy variable, de 0,5 hasta 2,6 cabezas/ $/$ a $^{7}$. Los índices productivos de estos sistemas son bajos, con entore de vaquillonas a los 3 años, porcentajes de marcación que oscilan del 40 al 50\% y una producción de carne de 80 a $100 \mathrm{~kg} / \mathrm{ha} / \mathrm{año}$; por lo tanto los animales son terminados con 3 o más años de edad.

La demanda de carne bovina de la Provincia de Misiones es creciente, el consumo alcanza $51.000 \mathrm{tn} /$ año siendo abastecida en solo el $23 \%$ por la producción de carne local, por lo cual casi el $70 \%$ del consumo anual es satisfecho con carne proveniente de otras provincias ${ }^{7}$. Los objetivos de producción no son bien definidos, existe una cultura productiva orientada hacia la vaca de cría como entendiéndose que la ganadería pasa exclusivamente por esta actividad ${ }^{7}$.

Sobre la base de las existencias ganaderas y su evolución en el tiempo se puede decir que existe una alta tenencia indebida de ganado que identifica una adicción a la tenencia con alta ineficiencia productiva y con objetivos de producción no conocidos, con desinformación manifiesta del potencial de producción de carne por el lado del ganado y de los ambientes posibles en donde esa carne es producida ${ }^{\mathrm{a}}$.

\footnotetext{
a Zehentner, G.O. Grupo foresto ganadero de Misiones. "El informativo". Año $1 \mathrm{~N}^{\mathrm{o}}$ 3. junio-julio. 2005.
}

La situación económica por la que está atravesando el sector ganadero condujo a los modelos de producción tradicionales a una severa crisis de rentabilidad que afecta su viabilidad futura. Esto crea la necesidad de generar modelos de producción más rentables y eficientes, en un marco de sustentabilidad ${ }^{18}$. Los sistemas silvopastoriles (SSP) surgen como una alternativa de producción para ser implementada por pequeños y medianos productores en un marco de sustentabilidad económica, social y ambiental ${ }^{10}$. El término sustentable implica que es repetible en el tiempo y hasta el momento es lo mejor conocido para la conservación de los recursos naturales (suelo, agua, clima) conformando un ambiente vital a la gente o sociedad cuyos reclamos ambientalistas son cada vez mayores y resulta necesario considerarlos.

Estos sistemas consisten en la implantación de especies forestales de rápido crecimiento en densidades de plantación menores a las convencionales, para obtener rentas a mediano y largo plazo por la producción de madera de alta calidad. Al mismo tiempo se trata de utilizar el potencial ganadero del terreno, para la obtención de beneficios económicos por la producción de carne, lana y leche. La combinación de las actividades productivas de especies forrajeras con arbóreas, elegidas adecuadamente, posibilita hacer más eficiente el uso de los recursos, generando un aumento en la productividad y sustentabilidad del sistema combinado.

Las interacciones entre los distintos componentes de un SSP (árbol-pastura-animal-suelo-agua) son dinámicas y complejas, con relaciones activas que generan cambios entre sus componentes y la necesidad de un manejo intensivo. El conocimiento de los diferentes grados de interacción y su manejo, son claves para la utilización racional y sustentable del sistema, siendo algunos de ellos, todavía una incógnita.

El Instituto Nacional de Tecnología Agropecuaria (INTA) ha realizado investigaciones sobre SSP en Misiones y en principio, ha demostrado su viabilidad productiva. En Cerro Azul, los módulos de ensayo en SSP han logrado producciones de carne entre 480 y $740 \mathrm{~kg} /$ ha/año, mientras que los productores líderes ligados a la institución superan los $300 \mathrm{~kg} / \mathrm{ha} / \mathrm{año}$ de carne; con respecto a la producción de madera obtuvieron $80 \mathrm{tn} / \mathrm{ha} / \mathrm{año}$ de madera libre de nudos de Pinus elliottii y más de 120 tn/ha/año de Pinus taeda. A las ventajas que presentan los SSP, se suman las condiciones ecológicas favorables de la provincia para la producción de madera y el crecimiento elevado de forrajes, los incentivos forestales y las tendencias de demanda de productos selectos reflejadas por los mercados, que se convierten en un conjunto de oportunidades para el desarrollo de estos sistemas en un marco de sostenibilidad y sustentabilidad ${ }^{9}$.

Por otro lado, aún no están claramente definidos los modelos y protocolo de producción para las diferentes zonas agroecológicas. En este sentido, la presente revisión realizada a partir de un estudio de casos sobre los sistemas silvopastoriles, validará la alternativa productiva de los mismos en modelos reales de producción 
de pequeños y medianos productores, y contribuirá a la generación de información relevante que describa el componente arbóreo-forrajero-ganadero en las diferentes combinaciones, brindando información técnica de los avances de resultados de la dinámica de los componentes que integran el sistema foresto-ganadero y las experiencias desarrolladas por productores líderes.

\section{MATERIAL Y MÉTODOS}

Mediante un estudio de casos se indagó el desarrollo de tres sistemas agroforestales del centro y norte de Misiones. Las experiencias se desarrollaron en tres parcelas ubicadas:

1) Departamento de Montecarlo paraje "Islas" sobre la ruta provincial $\mathrm{N}^{\circ} 15$ distante $18 \mathrm{~km}$ de la intersección con la ruta nacional $\mathrm{N}^{\mathrm{o}} 14$ y a $50 \mathrm{~km}$ de la ruta nacional $\mathrm{N}^{\circ} 12$, a $26^{\circ} 32^{\prime} 58.13^{\prime \prime} \mathrm{S}$ y $54^{\circ} 32^{\prime} 53.90^{\prime \prime} \mathrm{O}$;

2) Departamento Eldorado localidad 9 de Julio sobre la ruta provincial $\mathrm{N}^{\circ} 15$, a $26^{\circ} 38^{\prime} 03.87^{\prime \prime} \mathrm{S}$ y $54^{\circ} 22^{\prime}$ $56.63^{\prime \prime} \mathrm{O}$; y

3) Departamento San Ignacio, localidad Jardín América, Colonia Oasis distante a $15 \mathrm{~km}$ de la ruta nacional No 12 sobre el río Paraná, a $26^{\circ} 59^{\prime} 15.69^{\prime \prime} \mathrm{S}$ y $55^{\circ}$ $13^{\prime} 32.48^{\prime \prime} \mathrm{O}$.

Establecimientos 1 y 2. Estos dos SSP combinan una pastura megatérmica (Brachiaria brizantha cv Marandú) con Pinus híbrido F2 manejado intensivamente.

Área de estudio: los suelos son moderadamente profundos y pedregosos, clasificados taxonómicamente como Alfisoles con tala rasa previa de bosque primario degradado. El relieve es fuertemente ondulado a colinado (muy aptos para forestación y aptos a moderadamente aptos para cultivos perennes). Está regada por varios cursos de agua, el arroyo Piray Guazú y Piray Miní, existiendo manantiales que se utilizan para bebederos de los animales. Con respecto al clima, las diferencias que pueden establecerse dentro del territorio provincial no son apreciables. La estación más lluviosa fue la primavera, las precipitaciones oscilaron entre $1.700 \mathrm{a} 1.800 \mathrm{~mm}$. La temperatura media anual en el mismo sitio es de $20^{\circ} \mathrm{C}$. Las heladas se produjeron de mayo a agosto, pero las laderas de los cerros en general, están libres de ellas.

Desarrollo del SSP: el ensayo se realizó sobre un predio de 112 ha ubicadas en el Departamento Montecarlo, divididas en 4 potreros, donde ingresaron 249 novillos y 100 ha en Departamento Eldorado, divididas en 5 potreros de 20 ha cada uno, donde ingresaron 232 vaquillas. El componente animal ingresado fue Braford y Hereford, como categoría terneros a partir del segundo año de la implantación del pino; a partir del tercer año de implantado el pino fueron introducidos novillos, llevándose a cabo el engorde de los animales.

Se plantó el componente forestal, Pinus hibrido $F 2$, en el año 1999. La densidad inicial fue de 1.000 plantas/ha, con un espaciamiento de $5 \times 2 \mathrm{~m}$. Se practicaron podas sucesivas y se realizaron tres raleos. Este
SSP se implementó en campo a cielo abierto. De este componente forestal se analizaron las siguientes variables: diámetro a la altura de pecho $(\mathrm{DAP}-\mathrm{cm})$ (medida obtenida a una altura promedio de 1,3 m), incremento medio anual (DAP-cm/año), altura total promedio (HT-m), incremento medio anual (HT-m/año), altura de poda promedio (m), longitud de copa viva promedio/ árbol/m, longitud de copa viva/ha/m (medida que se toma desde la rama viva hasta el ápice).

El componente forrajero implantado en el año 2000 fue Brachiaria brizantha cv Marandu, por medio de siembra por semilla a una densidad de 5 a $6 \mathrm{~kg} / \mathrm{ha}$. Se analizaron variables como especie, implantación, producción de materia seca, porcentaje de proteína y energía en Mcal/kg MS.

Las actividades realizadas hasta la estabilización de estos dos SSP fueron: en el año 0 (1999) se preparó el suelo (monte degradado) mediante la tala de los árboles (apeo), empuje, amontonamiento de todas las ramas y troncos (formación de colleras) y eliminación por degradación; al año 1 (2000) se realizó la siembra de la especie forrajera, Brachiaria brizantha cv Marandu, a razón de 5 a $6 \mathrm{~kg} / \mathrm{ha}$. Al año 2 (2001) se permitió el ingreso de animales categoría terneros, cabe aclarar que el ingreso de los mismos debería realizarse a partir de los 3 a $4 \mathrm{~m}$ de altura de la especie forestal ya que en este caso se observaron plantas con daños físicos. Al año 3 (2002) se realizó la primera operación de poda en todos los ejemplares (poda baja) hasta 2 a 2,20 m de altura. En el año 4 (2003) se efectúo la segunda operación de poda (poda media) elevando a 3,50 a $4 \mathrm{~m}$ de altura. En este año se intervino con el primer raleo (selectivo). En el año 5 (2004) se hace la tercera operación de poda (poda alta) a 6,50 m, en la cual se podaron los árboles seleccionados (635 ejemplares). Se continuó con el pastoreo rotativo. En los años 6 (2005) y 7 (2006), se realizó la cuarta operación de poda hasta los $9 \mathrm{~m}$ de altura y un raleo para reducir la longitud de copa viva.

En el último año citado se ingresó con novillos en parcela Islas y vaquillas preñadas en la parcela 9 de Julio; la plantación alcanzaba $3.695 \mathrm{~m}$ lineales de copa viva, comprometiendo la entrada de luz, por lo que se realizó el raleo, quedando 270 árboles/ha en parcela 9 de Julio y 222 árboles/ha en parcela Islas.

De este ensayo se llevaron a cabo las mediciones sobre la producción de carne con las siguientes variables: categoría animal, biotipo, ganancia total y diaria, carga animal, caracterización de la especie forrajera (especie, implantación, producción de materia seca, porcentaje de proteína) y caracterización de las especies forestales (especie, longitud de copa, densidad de plantación).

Establecimiento 3. Este SSP combina la pastura Axonopus catarinensis var Valls con Pinus taeda manejado intensivamente.

Área de estudio: los suelos del lugar son rojo profundo (denominado de complejo 9), corresponden desde el punto de vista taxonómico a los Ultisoles, suelos de mejor aptitud agrícola y excelente aptitud para la ac- 
tividad forestal. El relieve es ondulado con lomas bien definidas y sectores inclinados con pendientes medias y cortas (20\%) hacia los cursos de agua representados por el río Paraná al este y el arroyo Tabay al norte, existiendo además una vertiente de donde se extrae el agua para los animales, poseyendo una cañería subterránea (de aproximadamente $4.000 \mathrm{~m}$ ) con agua de perforación. Las precipitaciones rondaron los $1900 \mathrm{~mm}$, la estación más lluviosa fue la primavera, mientras que las mayores deficiencias hídricas sucedieron en la primera mitad del verano. La temperatura media fue de $16^{\circ} \mathrm{C}$.

Desarrollo del SSP: El predio donde se llevó a cabo el ensayo cuenta con 140 ha divididas en 15 parcelas de 9 ha cada uno y 5 ha destinadas a instalaciones e infraestructura. Se plantó el componente forestal Pinus taeda, en el año 1998. La densidad inicial fue de 1.666 plantas/ha, con un espaciamiento de $3 \times 2 \mathrm{~m}$. La densidad inicial fue alta debido a que la idea principal era netamente forestal. Luego se decidió transformarlo en un SSP por lo que se realizó un manejo intensivo, debido a que se practicaron podas sucesivas (dos podas/ año) y se realizaron varios raleos. Esto significa que la implementación del SSP se realiza a partir de la "situación 3", de plantaciones de pinos a distintas edades. De este componente se midieron los mismos parámetros que en el caso anterior.

Se implantó el componente forrajero Axonopus catarinensis var Valls (pasto jesuita gigante), en el año 1999, en matas o guías a 1-3 m entre surcos y $1 \mathrm{~m}$ entre mudas. Fueron analizadas las mismas variables.

El componente animal, Braford, fue ingresado como categoría terneros en el segundo y tercer año de implantado el pino y luego, novillos a partir del cuarto año en adelante, llevándose a cabo el engorde de los animales. Se midieron las mismas variables que en el caso anterior.

Las actividades realizadas por año hasta la estabilización de este sistema silvopastoril fueron: en el año 0 (1998) se realizó la habilitación y preparación de suelo mediante el aplastamiento de pastizales, tala de los árboles (apeo) y picado del material leñoso, marcación y plantación de la especie forestal. En el año 1 (1999) se implantó la especie forrajera jesuita gigante. $\mathrm{Al}$ año 2 (2000) se realizó la primera operación de poda selectiva al $50 \%$ de los árboles (800 árboles/ha) hasta $1 \mathrm{~m}$ de altura, una segunda poda a una altura de $2 \mathrm{~m}$, y un raleo pre-comercial a los árboles no podados (apeo de 866 árboles). En el año 3 (2001) se realizó la tercera poda selectiva a $3 \mathrm{~m}$ de altura (500 árboles/ ha), una cuarta poda $4 \mathrm{~m}$ de altura (400 árboles/ha) e ingreso de los animales categoría terneros. Al año 4 (2002) se realizó el raleo comercial (400 árboles/ ha remanentes), la quinta operación de poda elevando a $5 \mathrm{~m}$ de altura $(400$ árboles) y sexta poda a $6 \mathrm{~m}$ de altura
(250 árboles). En el año 5 (2003) se realizó la séptima (7 $\mathrm{m}$ de altura a 250 árboles/ha) y octava ( $8 \mathrm{~m}$ de altura a 200 árboles/ha) operaciones de poda y se continuó con el pastoreo rotativo. En el año 6 (2004) se efectúo la novena poda (200 árboles/ha) 8,5 a $9 \mathrm{~m}$ de altura. $\mathrm{Al}$ año 7 (2005) se hizo un raleo comercial para reducir la longitud de copa viva (200 árboles/ha remanentes). En el año 8 (2006) quedando 168 árboles/ha remanentes, se hizo el ingreso de novillos y la medición de los parámetros del componente animal. En este sistema también se llevaron a cabo las mediciones respectivas a la producción de carne, caracterización de la especie forrajera y forestal.

\section{RESULTADOS Y DISCUSIÓN}

Los resultados obtenidos se detallan en las Tablas 1 (componente forestal), 2 (componente animal ) y 3 (componente forrajero).

Componente forestal. En Misiones es posible la obtención en turnos rollizos podados (libre de nudos) de 16 a 29 años, superiores a $40 \mathrm{~cm}$ bajo corteza en punta delgada, grado I (grado de mejor calidad), tanto latifoliadas como coníferas. El régimen silvícola (poda y raleo) se basa en el manejo necesario para generar las condiciones de radiación solar requeridas para el desarrollo de la pastura, que a través de raleos tempranos "pre-comerciales" y podas obliga a concentrar el crecimiento en los mejores árboles, con lo cual se tiende a maximizar la madera libre de nudos, es decir, madera de calidad ${ }^{12}$. En la Figura 1 se muestra la relación inversamente proporcional entre el rendimiento forrajero del pasto jesuita (tn/ha MS) y la longitud de copa (m) de Pinus taeda (parcela 3, Departamento San Ignacio).

Componente animal. Los SSP bajo estudio producen en promedio $359,66 \mathrm{~kg} / \mathrm{ha}$ en un año; esto es un incremento promedio por animal de $155,37 \mathrm{~kg}$ en un año. Estos datos coinciden con ensayos realizados en INTA Cerro Azul (Misiones), en los cuales en un período de 10 meses bajo las mismas condiciones de pasturas y

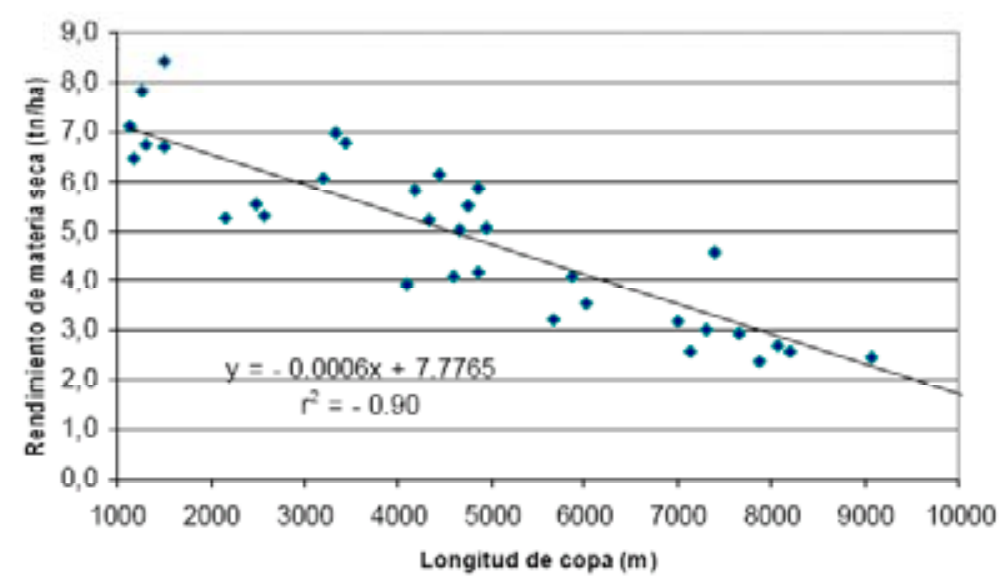

Figura 1. Relación entre la longitud de copa y el rendimiento forrajero de jesuita gigante bajo rodales de Pinus taeda de tres edades diferentes. 
Tabla 1. Análisis del componente forestal de los sistemas silvopastoriles estudiados.

\begin{tabular}{lccc}
\hline variables & $\begin{array}{c}\text { Departamento } \\
\text { Montecarlo }\end{array}$ & $\begin{array}{c}\text { Departamento } \\
\text { Eldorado }\end{array}$ & $\begin{array}{c}\text { Departamento } \\
\text { San Ignacio }\end{array}$ \\
\hline edad del pinal (años) & 10 & 10 & 10 \\
especie forestal & Pinus híbrido & Pinus híbrido & Pinus taeda \\
cantidad árboles/ha 3r. raleo & 270 & 222 & 168 \\
diámetro altura del pecho promedio (DAP cm) & 25,7 & 27,4 & 26,5 \\
incremento medio anual-IMA (cm/año) & 2,57 & 2,50 & 2,65 \\
altura total promedio-HT (m) & 18,09 & 21 & 17,3 \\
incremento medio anual-HT (m/año) & 1,80 & 1,90 & 1,73 \\
altura de poda promedio (m) & 9,28 & 9,40 & 7 \\
longitud de copa viva promedio/árbol/m. & 8,81 & 11,80 & 8 \\
longitud de copa viva/ha/m & 2.378 & 2.623 & 1.344 \\
\hline
\end{tabular}

Tabla 2. Análisis del componente animal de los sistemas silvopastoriles estudiados.

\begin{tabular}{lccc}
\hline variables & $\begin{array}{c}\text { Departamento } \\
\text { Montecarlo }\end{array}$ & $\begin{array}{c}\text { Departamento } \\
\text { Eldorado }\end{array}$ & $\begin{array}{c}\text { Departamento } \\
\text { San Ignacio }\end{array}$ \\
\hline superficie $(\mathrm{ha})$ & 100 & 112 & 140 \\
cantidad cabezas & 287 & 249 & 300 \\
categoría & vaquillas & novillos & novillos \\
biotipo & Braford, Hereford & Braford, Hereford & Braford \\
carga $($ animal $/ \mathrm{ha})$ & 2,87 & 2,22 & 2,14 \\
peso inicial $(\mathrm{kg})$ & 125,5 & 202,5 & 185,0 \\
peso final $(\mathrm{kg})$ & 280 & 349 & 350 \\
carga inicial $(\mathrm{kg} / \mathrm{ha})$ & 360 & 450 & 396 \\
unidad ganadera/ha & 0,90 & 1,12 & 0,99 \\
carga final $(\mathrm{kg} / \mathrm{ha})$ & 649 & 776 & 750 \\
unidad ganadera/ha & 1,62 & 1,94 & 1,87 \\
días transcurridos & 395 & 245 & 365 \\
producción $(\mathrm{kg} / \mathrm{ha})$ & 399,4 & 326,0 & 353,6 \\
ganancia peso $(\mathrm{g} / \mathrm{día})$ & 391 & 598 & 452 \\
\hline
\end{tabular}

Tabla 3. Análisis del componente forrajero de los sistemas silvopastoriles estudiados.

\begin{tabular}{lcc}
\hline variables & $\begin{array}{c}\text { Departamentos } \\
\text { Montecarlo y Eldorado }\end{array}$ & $\begin{array}{c}\text { Departamento } \\
\text { San Ignacio }\end{array}$ \\
\hline especie & $\begin{array}{c}\text { Brachiaria brizantha cv Marandú } \\
\text { (pasto brachiaria) }\end{array}$ & $\begin{array}{c}\text { Axonopus catarinensis Valls } \\
\text { (pasto jesuita gigante) }\end{array}$ \\
implantación & siembra $5-6 \mathrm{~kg}$ & plantación en matas a $1-3 \mathrm{~m}$ entre surcos y \\
& de semilla/ha $\mathrm{m}$ entre mudas.
\end{tabular}

raza ganadera, cada animal mostró un incremento de $151 \mathrm{~kg}$. Estos valores indican que estos SSP cumplen con un promedio de producción aceptado bajo las mismas condiciones de pastura y rodeo.

Debe destacarse que bajo dosel se pueden manejar los rodeos bovinos con alta proporción de sangre británica debido a que los árboles contribuyen a reducir el estrés calórico, con lo cual se logran mayores tasas de crecimiento y se puede aumentar la carga debido al aumento en la receptividad de las pasturas ${ }^{11,12,14,17 .}$. Sobre pastizal natural, bajo el dosel mencionado, fue posible obtener ganancias de peso promedio anual del orden $0,4-0,5 \mathrm{~kg} / \mathrm{animal} / \mathrm{día}$.; de esta manera se logran novillos pesados en promedio a los 27 meses de edad y por lo tanto esta región puede y tiene la posibilidad de pasar de ser zona de cría a zona de engorde.

Los resultados obtenidos de la evaluación durante cuatro años en módulos de engorde de novillos en sistemas pastoriles y SSP bajo dosel arbóreo de Pinus elliottii en Cerro Azul, si bien, las ganancias diarias de peso y producción de carne por hectárea tendieron a ser mayores en los módulos a cielo abierto, no registraron diferencias significativas $(\mathrm{p} \geq 0,005)$ en la comparación de medias a favor de los sistemas pastoriles en el promedio de los cuatro ciclos de evaluación.

En el ensayo estudiado, los resultados obtenidos respecto a las ganancias diarias de peso vivo y producción de carne por hectárea mostraron valores similares 
en los sistemas pastoriles y silvopastoriles ${ }^{15}$. Estos resultados coinciden con los descriptos en las parcelas de Eldorado, Montecarlo y San Ignacio. De acuerdo con el trabajo realizado en Cerro Azul, las diferencias con un sistema a cielo abierto son muy pequeñas, pero es importante destacar que los SSP presentan una ventaja económica sobre los pastoriles si se considera el retorno económico de la producción de carne y madera combinadas.

Componente forrajero. Al analizar las variables del componente forrajero en un SSP, se observa que existe una relación entre cantidad de $\mathrm{MS}(\mathrm{kg} / \mathrm{ha})$ que producen las forrajeras con los diferentes niveles de iluminación. En el mismo, se puede deducir que la cantidad mínima de radiación compatible con una producción aceptable de forraje se aproxima al 50\%. Brachiaria brizanta y pasto jesuita tienen mayor producción de MS/ha con un nivel de iluminación entre el 46 al 57\% ${ }^{1}$. Brachiaria brizanta es una gramínea perenne que en SSP crece bien y resiste mejor los efectos del frío y la sequía. Autores han encontrado que bajo pino produce por año hasta $6.000 \mathrm{~kg}$ de $\mathrm{MS} / \mathrm{ha}{ }^{19}$; dicho valor también se ha obtenido en las parcelas de estudio. Una serie de trabajos comunican que para obtener un buen rendimiento de materia seca por hectárea la necesidad mínima de luz de la pastura debe ser del $50 \%{ }^{4,12,14,17}$.

Por otra parte, Axonopus catarinensis var Valls (pasto jesuita gigante) es una pastura perenne que se destaca por tener alta calidad, crecimiento invernal y buena palatabilidad, características que la convierten en una de las mejores opciones para instalar en SSP que cuenten con suelos de buena calidad. Se ha demostrado que en SSP bajo pino produce por año entre 6.000 y $9.000 \mathrm{~kg} / \mathrm{MS} / \mathrm{ha}$ de forraje ${ }^{19}$. En las parcelas de estudio, se verificó que produce en promedio 8.000 $\mathrm{kg} / \mathrm{MS} / \mathrm{ha}$. Esta pastura presenta diferentes respuestas según la disponibilidad de los recursos luz y agua; se observó que a mayor nivel de sombra la fracción hoja se incrementó en todos los tratamientos evaluados (100, 70,50 y $30 \%$ de nivel de luz) y los componentes tallo y raíz disminuyeron. Alcanza máximos de producción con niveles de sombra del orden del 30 a $50 \%{ }^{13}$.

Con respecto al porcentaje de proteína bruta, podemos decir que se observa una tendencia de incremento de su contenido con la disminución del nivel de luz; siendo importante destacar que para Brachiaria brizantha cv Marandú (pasto brachiaria), dicho porcentaje en campos a cielo abierto es del $6 \%{ }^{16}$. Los antecedentes en cuanto a calidad son contradictorios, ya que se han observado aumentos en la digestibilidad con una reducción en el contenido de fibra detergente neutro ${ }^{8}$, ${ }^{20}$, en tanto que otros informan resultados opuestos ${ }^{21,}$ ${ }^{22}$. El aumento de la concentración de proteína bruta bajo sombra es el resultado más consistente entre los trabajos respecto a calidad ${ }^{3,8,22}$. Se ha reportado que la respuesta de los cultivares a los distintos niveles de luz es variable para el rendimiento, mientras que la calidad se favorece por el efecto de sombra, debido a un incremento del porcentaje de proteína ${ }^{1}$.

Colofón. Es importante destacar que los SSP son unidades complejas de producción, en las cuales se debe conocer el comportamiento de cada uno de sus componentes (forestal, animal y forrajero) y las interacciones entre ellos. Por tal motivo, es fundamental la capacitación del productor y la asistencia financiera para evitar los fracasos. Los servicios que brinda cada componente hacia los otros en SSP correctamente manejados, no generan situaciones de competitividad entre sí e incrementan la productividad total por unidad de superficie. Los SSP requieren un manejo tanto intensivo como integrador de sus componentes.

Mediante este estudio de casos surge que la longitud de copa demostró ser la variable más útil para el manejo forestoganadero integrado. En cuanto a la producción de carne, si bien las ganancias diarias de peso y producción de carne por hectárea tendieron a ser mayores en los módulos a cielo abierto, no hubo diferencias significativas entre ambos sistemas. La respuesta de las forrajeras a la variación de luz fue variable para el rendimiento, mientras que la calidad se favoreció por el efecto de sombra, debido a un incremento del porcentaje de proteína.

Los resultados emergentes de esta revisión bibliográfica demuestran que los SSP representan una alternativa viable para generar modelos productivos más rentables y eficientes en un marco de sustentabilidad.

\section{REFERENCIAS}

1. Benvenutti MA, Pavetti DR, Correa M, Perego JL. 2000. Evaluación de especies forrajeras gramíneas tropicales en distintos niveles de iluminación bajo monte forestal de pino para uso en sistemas forestoganaderos. Informe Técnico $N^{o} 79$, INTA EEA Cerro Azul, Misiones (Argentina), p. 18.

2. Casas RR, Rey Leyes MN, Abelardo S, Guanes AO, Lacorte SM, Capurro R. 1989. Erosión - Provincia de Misiones. En: El deterioro del ambiente en la Argentina, Ed FECIC, Buenos Aires, p. 130-135.

3. Eriksen FI, Whitney AS. 1981. Effects of light intensity on growth of some tropical forage species. I. Interaction of light intensity and nitrogen fertilization on six forage grasses. J. Agron 73: 427-433.

4. Fassola LE, Lacorte SM, Pachas N, Pezzuti R. 2005. Factores que influencian la producción de biomasa forrajera de Axonopus jesuiticus Valls, bajo dosel de Pinus taeda L. en el nordeste de Corrientes. RIA 34: 21-38.

5. Gunther DF, Correa M, Lysiak E. 2008. Zonas agroeconómicas homogéneas y sistemas de producción predominantes de la Provincia de Misiones. Boletín INTA EEA Cerro Azul, Misiones (Argentina), 9: 87.

6. Instituto Nacional de Estadística y Censos. 2003. Censo Nacional Agropecuario 2002. Resultados Generales. Ed. INDEC, Buenos Aires, $322 \mathrm{p}$. 
7. Instituto Nacional de Tecnología Agropecuaria - INTA. Plan de Tecnología Regional 2006-2008, Centro Regional Misiones, Posadas, p. 48.

8. Kephart KD, Buxton DR, Taylor SE. 1992. Growth of $\mathrm{C} 3$ and $\mathrm{C} 4$ perennial grasses under reduced irradiance. Crop Science 32: 1033-1038.

9. Kurtz VD, Pavetti DR. 2006. Sistema forestoganaderos con especies de rápido crecimiento (Pinus sp y Eucalyptus sp). Actas XXI Jorn Forestales Entre Ríos, Concordia, http://www.inta.gov.ar/concordia/capacita/jor_forestales/ jor_forestales.htm.

10. Kurtz VD, Khönke W. 2009. El asociativismo como estrategia para la implementación de los sistemas silvopastoriles en la Provincia de Misiones, Argentina. Actas I Congreso Nacional de Sistemas Silvopastoriles, Posadas (Misiones, Argentina).

11. Lacorte SM, Fassola LE, Pachas N, Colcombet L. 2004. Efectos de diferentes grados de sombreado con y sin fertilización fosfórica, sobre la producción de un pastizal modificado con predominio de Axonopus compressus (Swartz) Beauv en el sur de Misiones, Argentina. Actas XI Jornadas Técnicas Forestales y Ambientales, INTA Montecarlo (Misiones, Argentina), p. 9.

12. Lacorte SM, Esquivel JL. 2009. Sistemas silvopastoriles en la mesopotamia argentina. Reseña del conocimiento, desarrollo y grado de adopción. Actas I Congreso Nacional de Sistemas Silvopastoriles, Posadas (Misiones, Argentina).

13. Pachas AN, Fassola HE, Lacorte SM. 2004. Asignación de biomasa aérea y radical en plantas de Axonopus catarinensis bajo diferentes niveles de luz y agua. Boletín EEA INTA Montecarlo (Misiones, Argentina).

14. Pavetti DR, Benvenutti MA, Peruchena CO, Gunther DF, Correa M, Temchuk E. 1999. Alternativas de producción intensiva de carne bajo sistemas ganaderos y forestogana- deros en Misiones (Argentina). Informe de Avance, INTA EEA Cerro Azul (Misiones, Argentina), p. 18.

15. Pavetti DR, Benvenutti MA, Rossner MB. 2009. Sistemas pastoriles y silvopastoriles: comparación de su potencial productivo en Misiones (Argentina). Actas I Congreso Nacional de Sistemas Silvopastoriles, Posadas (Misiones, Argentina).

16. Perego JL. 1999. Brachiaria brizantha: implantación, manejo y producción. On line: http://www.inta.gov.ar/mercedes/info/publicaciones/bachiariabrizantha.htm.

17. Peri P. 1999. Efecto de la sombra sobre la producción y calidad de pasturas en sistemas silvopastoriles. SAGPYA Forestal (Buenos Aires) 13: 17-27.

18. Peruchena CO, Sampedro D. 1996. Algunas consideraciones sobre la suplementación de bovinos en pastoreo en el subtrópico. INTA EEA Mercedes (Corrientes, Argentina). Noticias y Comentarios $\mathrm{N}^{\circ} 309$, p. 5.

19. Rossner MB, Houriert JL, Pavetti DR. 2008. Descripción de las pasturas evaluadas en sistemas silvopastoriles del centro sur de la Provincia de Misiones. EEA Cerro Azul (Misiones, Argentina), Miscelánea $N^{\circ}$ 60, p. 32.

20. Samarakoon SP, Wilson JR, Shetlton HM. 1990. Growth, morphology and nutritive quality of shaded Stenotaphrum secondatum, Axonopus compressus and Pennisetum clandestinum. J Agric Sci 114: 161-169.

21. Wilson JR. 1982. Environmental and nutritional factors affecting herbage quality. In: Nutritional limits to animal production from pastures (Hacker JB Ed), CAB, Farnham Royal, p. 111-131.

22. Wilson JR, Wong CC. 1982. Effects of shade on some factors influencing nutritive quality of green panic and siratro pastures. Austr J Agric Res 33: 937-950. 